\title{
Post-Fabrication Modification of Forward Osmosis Membranes with a Poly(ethylene glycol) Block Copolymer for Improved Organic Fouling Resistance
}

\author{
Journal of Membrane Science
}

Revised: April 20, 2015

Devin L. Shaffer ${ }^{\mathrm{a}}$, Humberto Jaramillo ${ }^{\mathrm{a}}$, Santiago Romero-Vargas Castrillón ${ }^{\mathrm{a}}$, Xinglin $\mathrm{Lu}^{\mathrm{b}}$, and Menachem Elimelech ${ }^{\mathrm{a} *}$

${ }^{a}$ Department of Chemical \& Environmental Engineering, Yale University

New Haven, Connecticut 06520-8286, USA

${ }^{b}$ State Key Laboratory of Urban Water Resource and Environment Harbin Institute of Technology, Harbin, 150090, China

*Corresponding author.

email: menachem.elimelech@yale.edu; Tel. +1 (203) 432-2789; Fax +1 (203) 432-2881 


\begin{abstract}
Facile and effective strategies are needed to modify forward osmosis (FO) membranes for improved resistance to organic fouling. Fouling resistant FO membranes will advance the commercial implementation of FO for treating feed waters with high fouling potential, such as wastewater and brines. We report a membrane modification technique for post-fabrication grafting of a poly(ethylene glycol) (PEG) block copolymer to the surface of commercial thinfilm composite (TFC) FO membranes via an amide coupling reaction. The PEG concentration for membrane modification is optimized based on increased membrane hydrophilicity and reduced water permeability that result from increasing PEG concentrations during modification. Modified membranes exhibit improved resistance to organic fouling compared to unmodified control membranes when exposed to an aggressive synthetic wastewater mixture. The fouling resistance is achieved despite the non-uniform grafting of PEG, which is attributed to the limited accessibility of carboxylic group binding sites on the membrane surface. The fouling resistance of membranes modified using this post-fabrication technique compares favorably to TFC-FO membranes modified using other procedures. The modification technique we report in this work has the advantages of being relatively inexpensive, easy to implement, and applicable to commercial membranes.
\end{abstract}

Keywords: Forward osmosis, Membrane, Surface modification, Poly(ethylene glycol), Fouling

\title{
1 Introduction
}

Forward osmosis (FO) is an osmotically-driven membrane separation process that has promising applications in the treatment of feed waters with high fouling potential, such as wastewater and high-salinity brine [1]. Feed waters that are difficult to treat may in fact emerge as the niche commercial application for FO [2]. However, membrane fouling is a significant concern for the treatment of high-fouling potential waters by FO and for the more sustainable operation of FO without harsh cleaning agents or frequent membrane replacement. Although FO has demonstrated less fouling and more reversible fouling than reverse osmosis (RO) [3, 4], the polyamide thin-film composite (TFC) membranes that are the leading technology for FO are 
inherently prone to fouling because of their relative hydrophobicity, surface chemistry, and inherent surface roughness [5]. Reducing the fouling propensity of FO membranes is important to enable their expanded application, especially for feed waters with high fouling potential.

Surface modifications are an effective approach to reduce membrane fouling, and these modifications generally aim to attach hydrophilic polymer brushes or hydrophilic nanomaterials to the membrane surface to create a barrier to attachment of foulants. Poly(ethylene glycol) (PEG) is a well-studied and effective hydrophilic polymer used for surface modification [6] in techniques that are generally described as PEGylation. Membrane PEGylation imparts resistance to fouling by steric repulsion of foulants resulting from PEG polymer brush layer and the presence of bound water [7]. Depending on PEG polymer length and grafting density, the grafted PEG may form a brush-like layer or an entangled mushroom-like layer, but both systems have been shown to be effective at reducing adsorption of foulants to model surfaces [8].

PEGylation is a well-established technique in the biomedical field [9] and for drug delivery systems [10], and it has also been applied to membranes for water treatment. Several different PEGylation methods have been explored for membrane modification both during and after membrane fabrication. PEG has been incorporated into the membrane fabrication process by grafting amine-terminated PEG derivatives to acyl chloride groups in the nascent polyamide layer immediately following interfacial polymerization [11, 12]. The resulting modified membranes experienced less flux decline when exposed to feed solutions with model foulants. Enrichment of the membrane surface with amino functional groups during interfacial polymerization [13], and amino enrichment followed by PEG grafting [14], have also been demonstrated to reduce membrane organic fouling.

After fabrication, PEG modification of commercial RO membranes has been accomplished by coating the membrane surface with a crosslinked PEG hydrogel [15] and grafting PEG derivatives to the available functional groups on the membrane active layer [16]. Redox-initiated grafting of hydrophilic polymers has also been demonstrated for commercial polyamide RO membranes $[17,18]$. Other post-fabrication PEGylation techniques for modification of RO membranes include plasma polymerization of PEG derivatives [19] and initiated polymerization of antifouling monomers [20, 21]. 
Despite the advances in techniques for membrane surface modification with PEG, there remains a need for a facile, non-destructive, post-fabrication modification technique that is specifically tailored to FO membranes. Very few studies have focused on the specific challenge of FO membrane surface modification for organic fouling resistance, and advances in this area of research are important for FO treatment of waters with high fouling potential. Studies focusing on membrane modification and fouling behavior in FO rather than RO are important because different behavior is expected for FO compared to RO, as shown in previous fouling studies [3].

We investigated a facile, post-fabrication modification technique to graft Jeffamine, an inexpensive poly(ethylene glycol) (PEG) block copolymer, to the surface of commercial FO membranes to improve their resistance to organic fouling. The grafting technique relies upon a mediated coupling to bind the PEG block copolymer to native carboxylic groups on the FO membrane surface. The coupling technique was developed for protein complexation [22, 23] and has subsequently been demonstrated for binding nanomaterials [24, 25], PEG derivatives [26], and antibacterial enzymes [27] to the surfaces of polyamide membranes after fabrication. Jeffamine has been used previously for post-fabrication modification of RO membranes [26], but modification conditions were not optimized, fouling conditions representative of high fouling potential feed waters were not thoroughly investigated, and FO membranes and operating conditions were not explored.

In this work, we demonstrate the successful post-fabrication modification of commercial TFC-FO membranes with Jeffamine for improved organic fouling resistance. Through extensive membrane characterizations, we identify the optimal concentration for Jeffamine grafting. We report the results of membrane fouling tests employing an aggressive mixture of organic foulants, which demonstrate that Jeffamine-modified FO membranes experience less organic fouling than unmodified membranes. The fouling resistance of Jeffamine-modified membranes compares favorably to FO membranes modified using more intensive methods. Our work shows that post-fabrication Jeffamine modification is a facile and effective technique to improve membrane organic fouling resistance. 


\section{Materials and methods}

\subsection{Forward osmosis membranes}

Polyamide, thin-film composite, forward osmosis (TFC-FO) membranes were supplied by Oasys Water, Inc. (Boston, Massachusetts, USA). Membranes were moist when received and were stored in deionized (DI) water (Milli-Q, Millipore) and refrigerated at $4{ }^{\circ} \mathrm{C}$. Before use, membranes were wet by immersing in a $25 \%$ isopropanol solution for 30 minutes, followed by three 1 -h periods of soaking in DI water.

\subsection{Membrane surface modification with poly(ethylene glycol) block copolymer}

The surfaces of TFC-FO membranes were modified by the attachment of O,O'-Bis(2aminopropyl) poly(propylene glycol)-block-poly(ethylene glycol)-block-poly(propylene glycol). This amine-terminated poly(ethylene glycol) block copolymer is known by the trade name Jeffamine (Jeffamine ED-2003, MW 2,000 $\mathrm{g} \mathrm{mol}^{-1}$, Aldrich), which is registered trademark of the Huntsman Corporation. Jeffamine polymer chains were bound to the membrane surface using a carbodiimide-mediated, amide coupling reaction that consisted of two steps, as shown in Figure 1. As illustrated in Figure 1, Jeffamine ED-2003 consists of an estimated three repeating units of propylene glycol on each end of 39 repeating units of ethylene glycol [28].

For the modification procedure, the membranes were clamped onto plastic frames such that the active layer was isolated from the support layer. In the first step of the modification, an activation solution containing $2 \mathrm{mM} N$-(3-Dimethylaminopropyl)- $N$ '-ethylcarbodiimide (EDC, Thermo Scientific) crosslinker and $5 \mathrm{mM} \mathrm{N}$-Hydroxysuccinimide (NHS, Aldrich) was pipetted over the isolated active layer of the TFC-FO membrane. The activation solution was prepared in DI water buffered at pH 5 with $10 \mathrm{mM}$ 2-(N-Morpholino) ethanesulfonic acid (MES, Sigma) and containing $0.5 \mathrm{M}$ sodium chloride ( $\mathrm{NaCl}, \mathrm{J} . \mathrm{T}$. Baker). EDC in the activation solution reacts with carboxylic groups to form an unstable acylurea intermediate, which then reacts with NHS to form a more stable ester [23, 29]. After 10 minutes contact time, the activation solution was removed. Membrane active layers were rinsed with DI water, and $50 \mathrm{~mL}$ of Jeffamine solution was pipetted over the active layer. Jeffamine solutions (wt \%) were prepared at concentrations of $1 \%, 2 \%, 5 \%, 10 \%$, and $15 \%$ by dissolving Jeffamine in DI water containing $0.15 \mathrm{M} \mathrm{NaCl}$ and 
buffered to $\mathrm{pH} 7.5$ with $10 \mathrm{mM}$ 4-(2-Hydroxyethyl) piperazine-1-ethanesulfonic acid (HEPES, Sigma). The amine-terminated Jeffamine polymer forms an amide bond to the membrane surface by nucleophilic substitution of the NHS ester. Figure 1 illustrates Jeffamine attachment via a single amine terminus, but attachment of both ends of the polymer chain may also occur. After 10 minutes contact with the membrane surface, the Jeffamine solution was discarded, and the membrane active layer was rinsed with DI water followed by a rinse with $25 \%$ isopropanol. After a final DI water rinse, the Jeffamine-modified membranes were stored submerged in DI water in opaque plastic bottles refrigerated at $4{ }^{\circ} \mathrm{C}$.

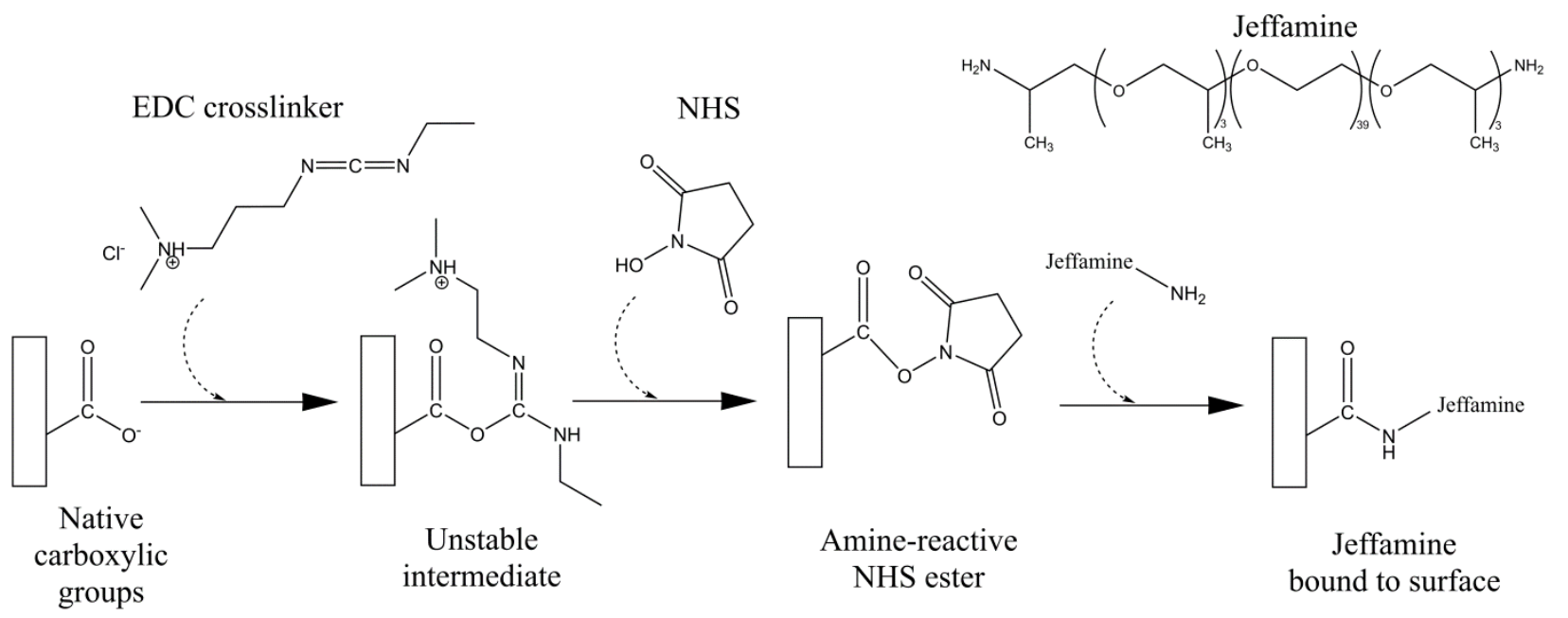

Fig. 1. Scheme illustrating Jeffamine binding to the membrane surface. Native carboxylic groups on the surface of polyamide thin-film composite membranes are activated by a solution containing $N$-(3Dimethylaminopropyl)- $N$ '-ethylcarbodiimide (EDC) and $N$-Hydroxysuccinimide (NHS).

Introduction of a solution containing amine-terminated Jeffamine, a poly(ethylene glycol) and poly(propylene glycol) block copolymer, results in nucleophilic substitution of the NHS ester and the formation of an amide bond between the Jeffamine polymer and the membrane surface.

\subsection{Characterization of membrane transport properties}

The water permeability coefficient $\left(A, \mathrm{~L} \mathrm{~m}^{-2} \mathrm{~h}^{-1} \mathrm{bar}^{-1}\right)$, salt permeability coefficient $\left(B, \mathrm{~L} \mathrm{~m}^{-2}\right.$ $\left.\mathrm{h}^{-1}\right)$, and salt rejection $(S R, \%)$ of the control and Jeffamine-modified membranes were determined using a bench-scale reverse osmosis membrane test unit. Permeate flux with a DI water feed and permeate flux and salt rejection with a $50 \mathrm{mM} \mathrm{NaCl}$ (J.T. Baker) feed solution 
were measured, as described in our previous publications [12, 14]. From these measurements, the $A$ coefficient was calculated from

$J_{w}=\boldsymbol{A} \Delta P$

where $J_{w}$ is the DI water permeate flux and $\Delta P$ is the applied hydraulic pressure of 28 bar (400 psi).

The $S R$ was calculated from

$S R=\left(1-\frac{C_{\text {permeate }}}{C_{\text {feed }}}\right)(100)$

where $C_{\text {feed }}$ is the feed solution concentration of $50 \mathrm{mM} \mathrm{NaCl}$ and $C_{\text {permeate }}$ is the permeate $\mathrm{NaCl}$ concentration. $C_{\text {feed }}$ and $C_{\text {permeate }}$ were measured using an $\mathrm{NaCl}$-calibrated electric conductivity meter (Oakton CON 110).

The $B$ coefficient was calculated from [30]

$B=\frac{J_{w, S}(1-S R)}{S R} \exp \left(-\frac{J_{w, s}}{k}\right)$

where $J_{w, s}$ is the permeate flux with $50 \mathrm{mM} \mathrm{NaCl}$ feed solution, and $k$ is the mass transfer coefficient for $\mathrm{NaCl}$ in the reverse osmosis test unit feed channel, approximated from a Sherwood correlation for laminar flow in the channel [30]. The mean values of three $J_{w, s}$ and $S R$ measurements were used for calculating the $B$ coefficient and $S R$ for each membrane tested, and 2-4 different membrane coupons were tested for each membrane modification condition.

\subsection{Characterization of membrane surface properties}

\subsubsection{Hydrophilicity by contact angle measurements}

Water contact angles were measured with a goniometer (VCA Video Contact Angle System, AST Products) using the sessile drop method. DI water droplets with a volume of $2 \mu \mathrm{L}$ were pipetted onto the surface of membrane coupons that had been air dried for a minimum of $36 \mathrm{~h}$ before measurements were collected. Each droplet was allowed to equilibrate with the membrane surface for $5 \mathrm{~s}$ before an image was captured with the goniometer video system. Image analysis software (VCA Optima XE) was used to calculate the left and right contact angles between the droplet and the membrane surface. Contact angle measurements were 
collected for 2-3 individual membrane coupons of the unmodified control and Jeffaminemodified membranes. A minimum of 36 contact angle measurements were recorded for each membrane coupon, resulting in a minimum of 72 contact angle measurements for each membrane modification condition.

\subsubsection{Charge density by TBO dye technique}

The density of negatively charged carboxylic groups on the surface of Jeffamine-modified membranes and unmodified control membranes was determined using a dye technique [31]. Toluidine Blue O dye (TBO, Sigma-Aldrich) was electrostatically bound to the surfaces of membrane samples at $\mathrm{pH} \mathrm{11,} \mathrm{when} \mathrm{the} \mathrm{surface} \mathrm{carboxylic} \mathrm{groups} \mathrm{were} \mathrm{deprotonated;} \mathrm{samples}$ were thoroughly rinsed at $\mathrm{pH} \mathrm{11;} \mathrm{and} \mathrm{dye} \mathrm{was} \mathrm{then} \mathrm{eluted} \mathrm{from} \mathrm{the} \mathrm{membrane} \mathrm{samples} \mathrm{at} \mathrm{pH} 2$, when the surface carboxylic groups were uncharged. Dye concentration was estimated by measuring ultraviolet light (UV) absorbance at $630 \mathrm{~nm}$ and comparing absorbance to a prepared calibration curve. The density of carboxylic groups on the membrane coupon surface was calculated from the dye concentration, elution solution volume, and area of the membrane coupon. TBO dye tests were performed on two samples from separately prepared batches of modified membranes and the corresponding unmodified control membranes. For each membrane sample subjected to dye testing, three separate membrane coupons were tested. UV absorbance measurements were collected for eight elution solution samples from each membrane coupon, resulting in 24 calculations of carboxylic group density for each membrane sample.

\subsubsection{Surface functionalization by ATR-FTIR spectroscopy}

Changes in surface chemistry of the Jeffamine-modified membrane surfaces were assessed by Fourier transform infrared (FTIR) spectroscopy with attenuated total reflectance (ATR). Membrane coupons were air dried for at least $24 \mathrm{~h}$ before spectra were measured on an FTIR spectrometer (Thermo Scientific Nicolet 6700) equipped with an ATR accessory consisting of a $\mathrm{ZnSe}$ plate $\left(45^{\circ}\right.$ angle of incidence) and a sample gripper device. Absorbance spectra were measured with 64 scans of each sample at a spectral resolution of $2 \mathrm{~cm}^{-1}$. The instrument was purged with nitrogen during measurements to reduce the interference of atmospheric moisture. 
Background measurements in air were collected before each membrane sample measurement. ATR-FTIR spectra were collected for two different membrane samples of each membrane modification condition.

\subsubsection{Surface chemistry by fluorescence imaging}

Jeffamine coverage on the surfaces of modified membranes was observed by fluorescence microscopy. Membranes were modified with a $2 \%$ Jeffamine solution that contained Rhodamine-labeled Jeffamine [12]. The Jeffamine was prepared by mixing $0.1 \mathrm{mmol}$ Jeffamine with $0.01 \mathrm{mmol}$ Rhodamine B isothiocyanate (Sigma-Aldrich) in $0.1 \mathrm{M}$ sodium carbonate $\left(\mathrm{Na}_{2} \mathrm{CO}_{3}\right.$, Sigma-Aldrich) buffer solution at $\mathrm{pH} 9$ and at room temperature for $4 \mathrm{~h}$. Following this reaction, the Rhodamine-labeled Jeffamine was dialyzed (Spectra/Por7 dialysis tubing, 1,000 Da molecular weight cut off) in 1 L DI water for 4 days to remove free dye molecules (DI water was changed three times per day). The Rhodamine-labeled Jeffamine was then freeze-dried and mixed with unlabeled Jeffamine at a mass ratio of 1:100 prior to preparing the $2 \%$ Jeffamine solution for membrane modification. Unmodified control membranes and membranes modified with Rhodamine-labeled Jeffamine at $2 \%$ concentration were imaged using a confocal microscope (Zeiss LSM 510, Carl Zeiss Group) with a fluorescence detector. Excitation was performed at $550 \mathrm{~nm}$ and emission was observed at $610 \mathrm{~nm}$. Four different membrane coupons modified with $2 \%$ Jeffamine solution were each imaged at four random locations, and extent of Rhodamine (red) fluorescence coverage for each image was determined using ImageJ software (release 1.46, imagej.nih.gov).

\subsubsection{Surface roughness by AFM}

Membrane surface roughness was measured by atomic force microscopy (AFM) in tapping mode (multi-mode AFM, Digital Instruments, Bruker Corporation). Membrane samples were dried in a desiccator for a minimum of $36 \mathrm{~h}$ before AFM measurements. Surface roughness measurements were collected with a silicon probe (Budget Sensors, Tap300AI-G, resonant frequency $=300 \mathrm{kHz}$ ) at a scan rate of $0.5 \mathrm{~Hz}$ over an area of $100 \mu \mathrm{m}^{2}$. Two randomly-chosen locations were scanned on each of two membrane coupons for both the control and Jeffamine- 
modified membranes. AFM data were analyzed using Nanoscope Controller Software (Version 5.31r1, Bruker Corporation) that calculated the root mean square roughness for each sample area.

\subsection{Organic fouling experiments in forward osmosis}

The fouling propensities of control and Jeffamine-modified membranes were assessed by dynamic fouling experiments in a bench-top FO membrane test unit using a high fouling potential feed solution. The specific details of the test unit configuration are described in our previous work [32]. Dynamic fouling experiments were conducted with a feed solution mimicking the ionic composition of synthetic secondary wastewater effluent (SWWE) from wastewater treatment facilities in California [33]: $0.94 \mathrm{mM}$ ammonium chloride $\left(\mathrm{NH}_{4} \mathrm{Cl}\right.$, Sigma), $0.45 \mathrm{mM}$ potassium phosphate $\left(\mathrm{KH}_{2} \mathrm{PO}_{4}, \mathrm{BDH}-\mathrm{VWR}\right), 0.5 \mathrm{mM}$ calcium chloride $\left(\mathrm{CaCl}_{2} \cdot 2 \mathrm{H}_{2} \mathrm{O}\right.$, Sigma-Aldrich), $0.5 \mathrm{mM}$ sodium bicarbonate $\left(\mathrm{NaHCO}_{3}\right.$, Sigma-Aldrich), $2.0 \mathrm{mM}$ $\mathrm{NaCl}$ (J.T. Baker), and $0.6 \mathrm{mM}$ magnesium sulfate $\left(\mathrm{MgSO}_{4} \cdot 7 \mathrm{H}_{2} \mathrm{O}\right.$, Sigma-Aldrich). The synthetic SWWE solution ionic strength was $14.6 \mathrm{mM}$, and the final solution $\mathrm{pH}$ was 7.4. Bovine serum albumin protein (BSA, Sigma), sodium alginate (Aldrich), and Suwannee River natural organic matter (SRNOM, International Humic Substances Society) were added to this feed solution as models of the proteins, polysaccharides, and organic matter present in SWWE.

High concentrations of these foulants $\left(100 \mathrm{mg} \mathrm{L}^{-1}\right.$ each) were used to create an aggressive mixture with which to assess the organic fouling potential of the membranes. Two sets of dynamic fouling experiments were conducted, one using $\mathrm{NaCl}$ as a draw solute and the other using magnesium chloride $\left(\mathrm{MgCl}_{2} \cdot 6 \mathrm{H}_{2} \mathrm{O}\right.$, J.T. Baker) as the draw solute, in order to study potential effects of reverse salt flux of draw solution on organic fouling. Two unmodified control membranes and three Jeffamine-modified membranes were tested with $\mathrm{NaCl}$ draw solution, and three unmodified control membranes and two Jeffamine-modified membranes were tested with $\mathrm{MgCl}_{2}$ draw solution.

For experiments with $\mathrm{NaCl}$ draw solution, the draw solution concentration was $\sim 1 \mathrm{M}$ but varied for each experiment in order to achieve initial baseline permeate water fluxes of $20.1 \pm 0.8 \mathrm{~L} \mathrm{~m}^{-2} \mathrm{~h}^{-1}$ (mean \pm standard deviation). For experiments with $\mathrm{MgCl}_{2}$ draw solution, the draw solution concentration varied from 1.0 to $1.5 \mathrm{M}$ with resulting initial baseline permeate 
water fluxes of $17.5 \pm 1.8 \mathrm{~L} \mathrm{~m}^{-2} \mathrm{~h}^{-1}$. Initial feed solution and draw solution volumes were $2 \mathrm{~L}$, and solution temperatures were maintained at $25 \pm 1{ }^{\circ} \mathrm{C}$. Feed and draw solutions were pumped in cocurrent flow across the membrane at a flowrate of $400 \mathrm{~mL} \mathrm{~min}^{-1}$, which resulted in a crossflow velocity of $\sim 8.5 \mathrm{~cm} \mathrm{~s}^{-1}$ and laminar flow conditions $(\operatorname{Re} \sim 500)$ in the feed and draw solution channels. Permeate flux was calculated from the change in draw solution weight over time, and experiments were conducted until $500 \mathrm{~mL}$ of permeate was collected ( 16 h duration).

Each dynamic fouling experiment comprised four parts: baseline, fouling, cleaning, and recovery. The baseline portion of the experiment was conducted with the SWWE feed solution ionic composition to determine the permeate flux decline that occurred because of draw solution dilution and feed solution concentration resulting from batch mode operation. During the fouling portion of the experiment, BSA, sodium alginate, and SRNOM were added to the SWWE feed solution at a concentration of $100 \mathrm{mg} \mathrm{L}^{-1}$ each. After the fouling portion of the experiment, the membrane was cleaned in place by a $15 \mathrm{mM} \mathrm{NaCl}$ cleaning solution pumped on the feed and draw solution sides of the membrane at an increased flowrate of $1 \mathrm{~L} \mathrm{~min}^{-1}\left(21 \mathrm{~cm} \mathrm{~s}^{-1}\right)$. Following cleaning, the irreversibility of membrane fouling was assessed through a recovery test, which repeated the conditions of the baseline portion of the experiment.

\subsection{Surface adhesion force measurements by AFM}

Atomic force microscopy (multi-mode AFM, Veeco Metrology Group, Bruker Corporation) was employed to measure adhesion forces between membrane surfaces and a model foulant probe. The foulant probe was prepared by gluing a $4-\mu \mathrm{m}$ diameter, carboxyl-modified, latex particle (Invitrogen Molecular Probes, Life Technologies) to the tip of a silicon nitride AFM cantilever (Bruker, NP-O10, spring constant $=0.06 \mathrm{~N} \mathrm{~m}^{-1}$ ) using optical adhesive (Optical Adhesive 68, Norland Products) cured by exposure to UV light, as described in our recent publication [14]. Before AFM measurements, the functionalized cantilever was immersed

overnight in $4 \mathrm{~g} \mathrm{~L}^{-1}$ sodium alginate solution at $4{ }^{\circ} \mathrm{C}$ to promote the adsorption of alginate to the probe so that its interactions with the membrane surface would better approximate the foulants present in SWWE.

Probe deflection versus piezo position measurements were collected in AFM contact mode for probe approach to and retraction from the membrane surface for three different samples each 
of control and Jeffamine-modified membranes. Measurements were conducted in a liquid cell containing SWWE solution. After soaking overnight in $4 \mathrm{~g} \mathrm{~L}^{-1}$ sodium alginate solution, the same functionalized cantilever was reused for force measurements of each membrane sample. Measurements were collected at seven random locations on each membrane sample, and each location yielded a minimum of seven usable measurements. A minimum of 145 measurements were collected for control and modified membranes. AFM data were analyzed using Nanoscope Analysis Software (Version 1.40, Bruker Corporation) to convert retraction data to force vs. separation curves and to identify the maximum adhesion force and associated distance for each probe retraction event.

\section{Results and discussion}

\subsection{Optimal Jeffamine concentration for surface modification}

A range of Jeffamine solution concentrations for membrane modification were studied to determine the optimal Jeffamine concentration that would improve membrane fouling resistance while limiting adverse impacts to membrane transport properties. The optimal Jeffamine concentration was determined by comparing changes in membrane water permeability at different Jeffamine modification concentrations to corresponding changes in membrane surface characteristics.

\subsubsection{Impact on membrane transport properties}

Jeffamine grafting reduced the membrane water permeability $(A)$ for all Jeffamine concentrations used in the membrane modification procedure. The membrane transport properties are summarized in Figure 2. Reductions in water permeability for modified membranes compared to the unmodified control are statistically significant (significance level $\alpha=0.05$ ) for $1 \%, 5 \%, 10 \%$, and $15 \%$ Jeffamine membranes, as determined by a two-sided t-test. The water permeability of modified membranes does not appear to trend with increasing Jeffamine concentration. The observed reduction in water permeability for modified membranes is attributed to an increased resistance to mass transport caused by the grafted Jeffamine layer. Similar reductions in water permeability or pure water flux have been observed for attachment of 
Jeffamine [26] and other PEG derivatives [16, 19] to commercial polyamide NF and RO membranes and to TFC-FO membranes $[12,14]$. Membrane salt permeability $(B)$ and salt rejection $(S R)$ were not significantly affected by Jeffamine grafting, and preservation of high salt rejection indicates that the grafting method does not damage the membrane active layer.

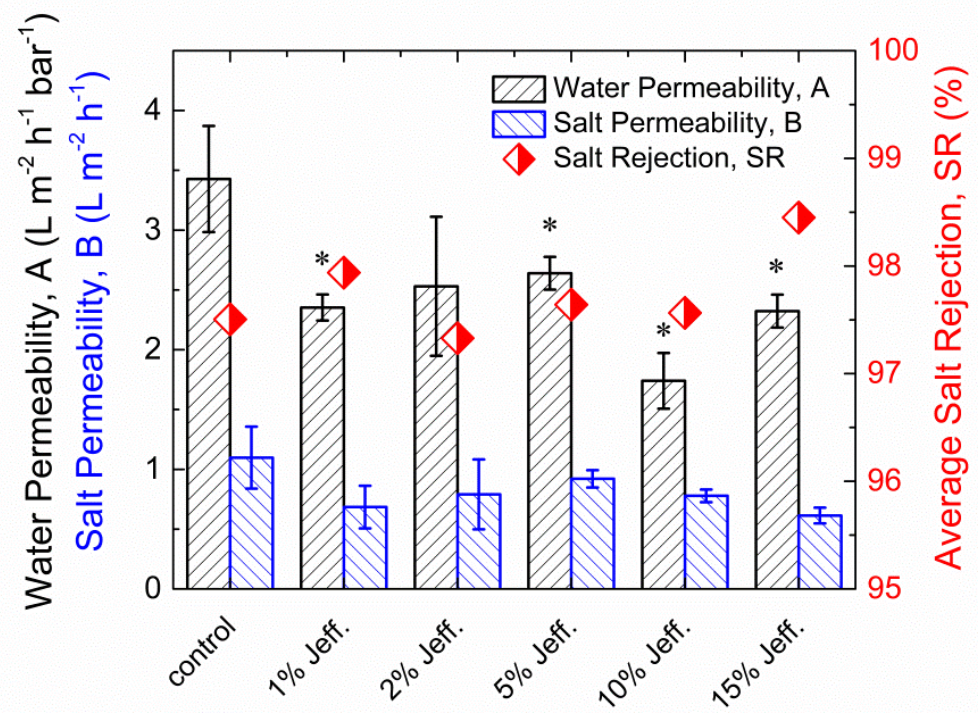

Fig. 2. Water permeability coefficients $\left(A, \mathrm{~L} \mathrm{~m}^{-2} \mathrm{~h}^{-1} \operatorname{bar}^{-1}\right)$, salt permeability coefficients $\left(B, \mathrm{~L} \mathrm{~m}^{-2}\right.$ $\mathrm{h}^{-1}$ ), and salt rejection $(S R, \%)$ of the control and Jeffamine (Jeff.)-modified membranes, as measured by reverse osmosis testing. Bars and symbols represent the mean of 2-4 separate tests of different membrane coupons for each membrane type. Error bars extend to one standard deviation. Significant differences between values for the Jeffamine-modified membranes compared to the unmodified control membranes are indicated by ' $*$ ' symbol and were determined in a two-sided ttest at a significance level $\alpha=0.05$.

\subsubsection{Impact on membrane surface characteristics}

\subsubsection{Hydrophilicity}

Changes in membrane surface hydrophilicity were assessed by water contact angle. Previous work on surface modifications for polyamide RO [19] and FO [12,14] membranes has demonstrated that reduced water contact angle is a reliable indicator of PEG attachment. More hydrophilic membrane surfaces have been correlated with improved membrane fouling 
resistance [6, 34]. Specifically, for TFC-FO membranes, recent work has demonstrated that hydrophilic surface modifications reduced the extent of organic fouling for modified membranes compared to unmodified controls $[12,14]$. Consequently, water contact angle measurements were used as an indicator of both Jeffamine grafting and associated membrane fouling resistance.

Significant reductions in water contact angle were observed for the Jeffamine-modified membranes compared to the unmodified control membranes (two-sided t-test, $\alpha=0.05$ ), suggesting an improvement in the fouling resistance of the modified membrane surfaces. The distributions of water contact angles measured for each membrane modification condition are shown in Figure 3. The mean water contact angle decreases with increasing Jeffamine concentration for membranes modified with $1 \%, 2 \%$, and $5 \%$ Jeffamine. However, further reductions in mean water contact angle are not observed for membranes modified with Jeffamine concentrations greater than 5\% Jeffamine.

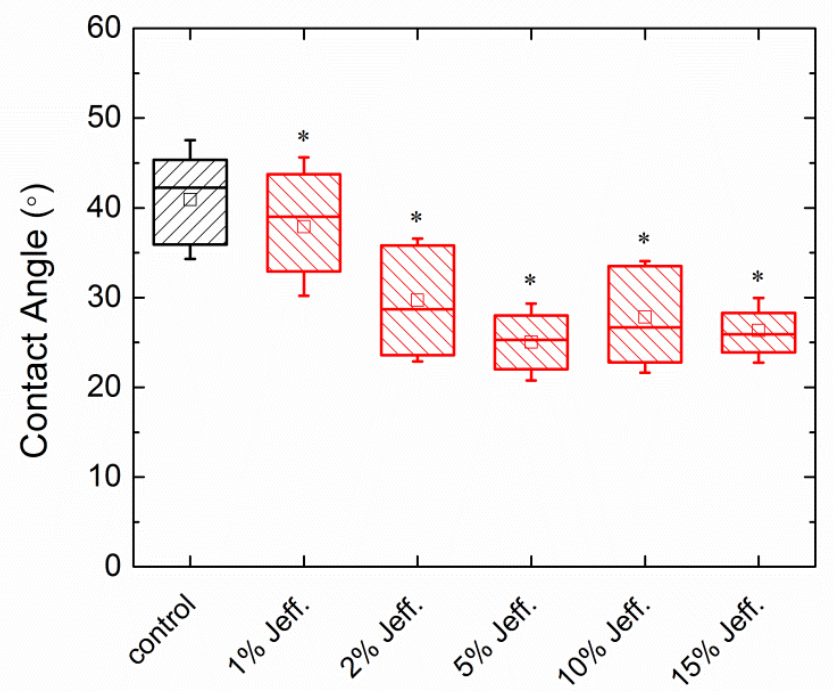

Fig. 3. Comparison of measured water contact angles for unmodified control membranes and membranes modified with different concentrations of Jeffamine (Jeff.) solution. Boxes represent the $25^{\text {th }}, 50^{\text {th }}$, and $75^{\text {th }}$ percentile values for a distribution of at least 72 contact angle measurements on 23 coupons of each membrane type. Whiskers extend to one standard deviation, and square symbols inside the boxes indicate the mean contact angle. The '*' symbol denotes that water contact angles for all Jeffamine-modified membranes are significantly less than the contact angles of the unmodified control membrane, as determined by two-sided t-tests at a significance level $\alpha=0.05$. 


\subsubsection{Degree of Jeffamine grafting}

ATR-FTIR spectra were measured for Jeffamine-modified membranes and compared to the spectra of unmodified control membranes as another qualitative indicator of Jeffamine attachment. Spectra show characteristic bands of the membrane polyamide active layer and the underlying polysulfone support layer due to the relatively large penetration depth of the IR [35]. The degree of grafting may be calculated from ATR-FTIR spectra as a parameter to facilitate the comparison of different membrane modification conditions. Degree of grafting is defined as the ratio of absorbance at a wavenumber indicative of the grafted polymer and absorbance at a wavenumber that is indicative only of the unmodified membrane and that is not affected by the presence of the modifying agent [18]. Spectral changes at wavenumber $1080 \mathrm{~cm}^{-1}$ are commonly used to assess PEG grafting to polyamide membranes and are attributed to the $\mathrm{C}-\mathrm{O}$ and $\mathrm{C}-\mathrm{C}$ stretch of poly(ethylene glycol) $[11,12,14,16,26]$. Accordingly, degrees of Jeffamine grafting were calculated from ATR-FTIR spectra as the ratio of Jeffamine absorbance at wavenumber $1080 \mathrm{~cm}^{-1}\left(A_{1080 \mathrm{~cm}-1}\right)$ to polyamide absorbance at reference wavenumber $1150 \mathrm{~cm}^{-1}\left(A_{1150 \mathrm{~cm}-1}\right)$ or $1170 \mathrm{~cm}^{-1}\left(A_{1170 \mathrm{~cm}-1}\right)$, which are attributed to symmetric $\mathrm{C}-\mathrm{SO}_{2}-\mathrm{C}$ stretching in the polysulfone support layer [36, 37].

Figure 4(A) includes representative ATR-FTIR spectra for an unmodified control TFC-FO membrane and a Jeffamine-modified membrane. Degrees of Jeffamine grafting calculated at both $1150 \mathrm{~cm}^{-1}$ and $1170 \mathrm{~cm}^{-1}$ reference wavenumbers are shown in Figure 4(B). Statistically significant increases in the calculated degrees of Jeffamine grafting (two-sided t-tests, $\alpha=0.05$ ) were observed for membranes modified at $2 \%, 5 \%, 10 \%$, and $15 \%$ Jeffamine compared to the unmodified control. The calculated degrees of grafting at 2\%-15\% Jeffamine are not statistically different, indicating no additional Jeffamine grafting to the membrane surface occurs at concentrations greater than $2 \%$ Jeffamine. 

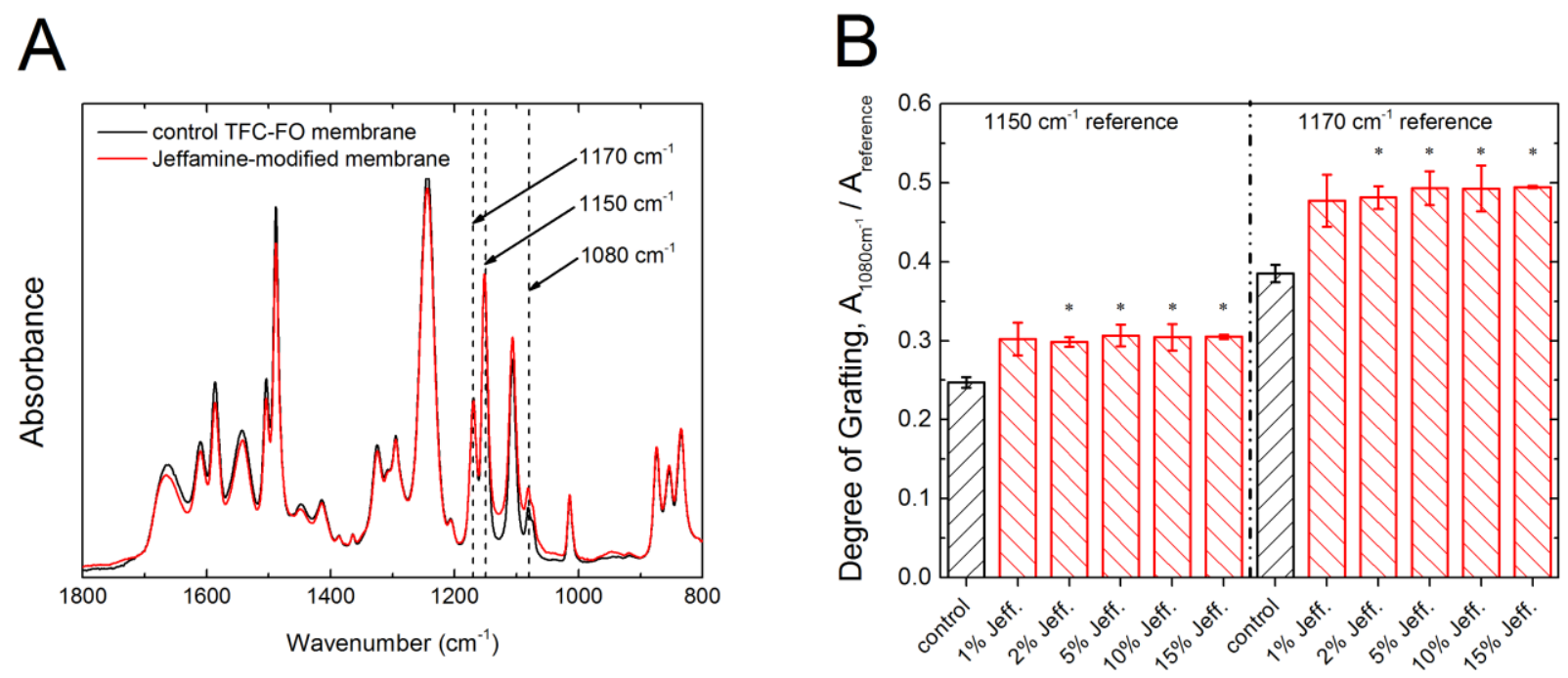

Fig. 4. A) Representative attenuated total reflectance-Fourier transform infrared (ATR-FTIR) absorbance spectra for an unmodified control thin-film composite forward osmosis (TFC-FO) membrane and a Jeffamine-modified membrane. Absorbance peaks at wavenumbers $1150 \mathrm{~cm}^{-1}$ and $1170 \mathrm{~cm}^{-1}$ are attributed to the membrane polysulfone support layer $\left(\mathrm{C}_{-}-\mathrm{SO}_{2}-\mathrm{C}\right.$ stretch$)$, and an increase in the absorbance peak at wavenumber $1080 \mathrm{~cm}^{-1}$ is attributed to the presence of grafted Jeffamine on the modified membrane surface (C-O and C-C stretch). B) Comparison of degrees of grafting for membranes modified at different Jeffamine (Jeff.) solution concentrations and unmodified control membranes. Degrees of grafting were calculated from ATR-FTIR spectra as the ratio of absorbance at $1080 \mathrm{~cm}^{-1}\left(A_{1080 \mathrm{~cm}-1}\right)$ to absorbance at either $1150 \mathrm{~cm}^{-1}$ or $1170 \mathrm{~cm}^{-1}\left(A_{\text {reference }}\right)$. Bars represent the mean degree of grafting calculated from spectra collected for two different membrane samples. Error bars extend to one standard deviation. Significant differences between the degree of grafting for Jeffamine-modified membranes compared to the unmodified control are indicated by a ${ }^{*}$, symbol and were assessed by a two-sided t-test at significance level $\alpha=0.05$.

\subsubsection{Charge density}

Successful Jeffamine binding to the membrane surface reduces the number of native carboxylic groups. Thus, reduced carboxylic group densities for the Jeffamine-modified membranes compared to the unmodified control membrane are attributed to the grafting of Jeffamine to this fraction of carboxylic groups. Figure 5 presents the results of two TBO dye tests quantifying the densities of carboxylic groups for a set of control, $2 \%$ Jeffamine-modified, and $15 \%$ Jeffamine-modified membranes. Compared to the unmodified control membranes, the mean reductions in calculated carboxylic group densities for the $2 \%$ Jeffamine membranes are 
$\sim 7 \%$ and $\sim 2 \%$. No difference in the carboxylic group surface densities is observed for membranes modified at $2 \%$ and $15 \%$ Jeffamine concentrations, further indicating that no additional Jeffamine grafting occurred for membrane modifications performed at concentrations greater than $2 \%$. The different reductions in calculated carboxylic group surface densities for the two sets of modified membranes shown in Figure 5 may be attributed to slight variations in the modification conditions and resulting degrees of modification for the separately-prepared sets of membranes. The difference may also be a result of non-specific dye adsorption during the TBO dye technique and the sensitivity of the calibration curve relating UV absorbance and TBO dye concentration [31].

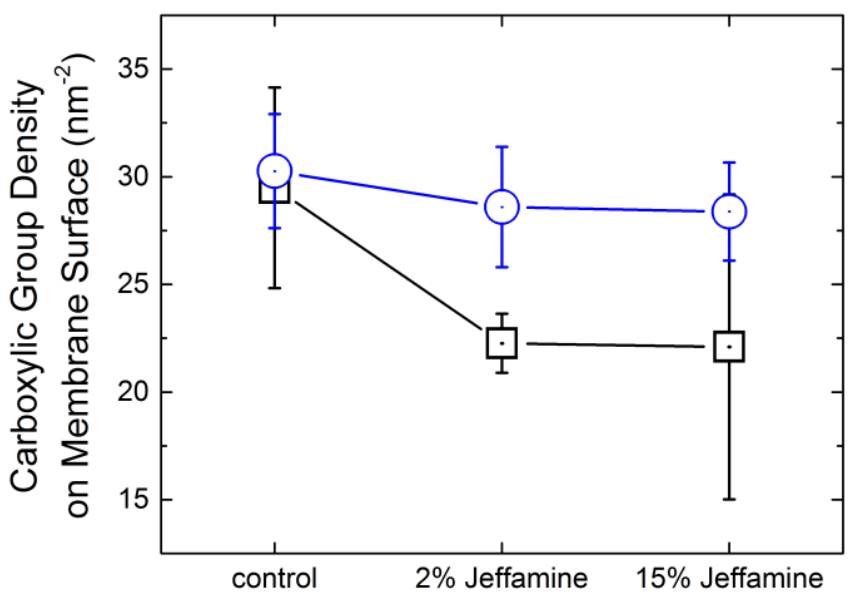

Fig. 5. Comparison of carboxylic group surface densities for control and Jeffamine-modified membranes, calculated from the results of Toluidine Blue O (TBO) electrostatic dye tests. Blue circles and black squares represent separate dye tests, each performed on samples of unmodified control membranes and membranes modified at $2 \%$ Jeffamine and $15 \%$ Jeffamine concentrations. Each test was conducted on three coupons of each membrane type, and eight measurements were collected for each membrane coupon. Symbols represent the mean carboxylic group density calculated from 24 measurements, and error bars extend to one standard deviation. 


\subsubsection{2\% Jeffamine as optimal concentration for membrane modifications}

Considering the transport properties and surface characteristics of the modified membranes discussed in the previous subsections, we identified $2 \%$ Jeffamine as the optimal solution concentration for membrane modification. A $2 \%$ Jeffamine solution concentration achieves the apparent maximum grafting of Jeffamine to the membrane surface and the associated maximum reduction in water contact angle. Membranes modified with 2\% Jeffamine demonstrate an acceptable reduction in water permeability compared to the unmodified control membrane and the membranes modified at other Jeffamine solution concentrations. Experiments investigating the organic fouling resistance of modified membranes were conducted with membranes modified with this optimal $2 \%$ Jeffamine concentration.

\subsection{Non-uniform Jeffamine grafting}

Transport properties and surface characteristics of the Jeffamine-modified membranes indicate a limit to the density of Jeffamine grafting using the EDC-NHS coupling technique. Surface charge density measurements show that carboxylic groups are still present on the membrane surface following the modification, although not all of these sites may be available for Jeffamine grafting. We further characterized the $2 \%$ Jeffamine-modified membranes to investigate the uniformity of grafting and to gain insight into the basis of the limitation to grafting uniformity and density.

\subsubsection{Confocal imaging of labeled Jeffamine membranes}

Confocal microscopy images of the surfaces of membranes modified with Rhodaminelabeled Jeffamine were analyzed and compared to those of unmodified control membranes. The confocal images demonstrate that Jeffamine attachment to the membrane surface is non-uniform with distinct micrometer-scale areas of Jeffamine on the membrane surface, as shown in the representative image in Figure 6A. The control membrane exhibited no fluorescence, confirming that the Rhodamine-labeled Jeffamine was the source of the red fluorescence for the modified membranes. For the 16 membrane images analyzed, the mean surface coverage of Rhodaminelabeled Jeffamine was less than $1 \%$ of the membrane area. 

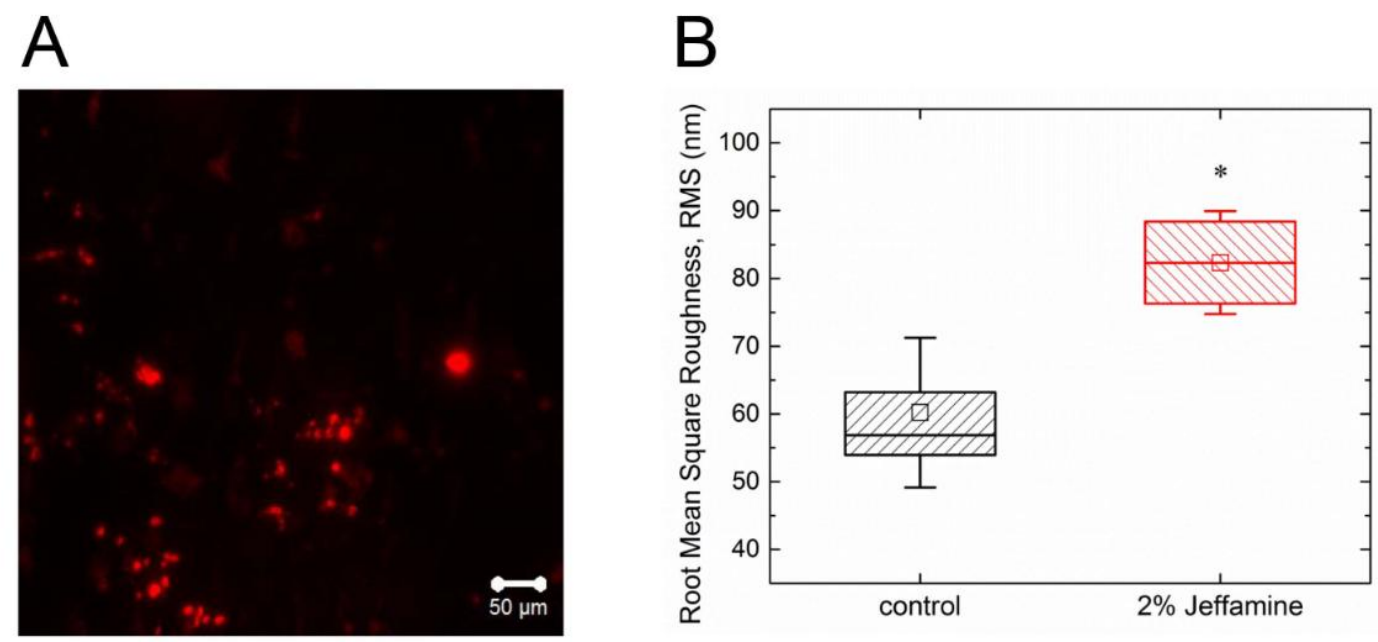

Fig. 6. Membrane characteristics indicating the non-uniform attachment of Jeffamine to the modified membrane surface. A) Confocal microscopy image of a representative membrane sample that was modified with Rhodamine-labeled Jeffamine solution at $2 \%$ concentration. Red fluorescence of the Rhodamine dye (excitation at $550 \mathrm{~nm}$ and emission observed at $610 \mathrm{~nm}$ ) demonstrates the non-uniform binding of Jeffamine to the membrane surface. B) Comparison of root mean square roughness $(R M S, \mathrm{~nm})$ for unmodified control membranes and membranes modified with $2 \%$ Jeffamine solution. Boxes represent the $25^{\text {th }}, 50^{\text {th }}$, and $75^{\text {th }}$ percentile values for a distribution of at least four RMS measurements on two coupons of each membrane type. Whiskers extend to one standard deviation, and square symbols inside the boxes indicate the mean sample $R M S$ roughness. The ' $*$ ' symbol denotes that $R M S$ roughness for $2 \%$ Jeffamine-modified membranes is significantly greater than the $R M S$ roughness of the unmodified control membranes, as determined by a two-sided t-test at a significance level $\alpha=0.05$.

Rhodamine-labeled Jeffamine was also used in our recent study [12] to examine the extent of Jeffamine coverage on membrane surfaces modified in situ during fabrication. The in situ modified membranes, for which Jeffamine was grafted to acyl chloride groups in the nascent polyamide layer, showed mean surface coverage by Rhodamine-labeled Jeffamine that was an order of magnitude greater than the Jeffamine-modified membranes presented in this work. The greater extent of Jeffamine coverage for the in situ modified membranes versus these postfabrication modified membranes suggests that a higher grafting density is achievable when Jeffamine is grafted to acyl chloride groups in situ rather than after membrane fabrication, at which point acyl chloride groups have been hydrolyzed to carboxylic groups, and EDC-NHS is necessary to mediate the Jeffamine grafting. 
Other studies have similarly observed low surface grafting density when PEGylating polyamide membranes using post-fabrication techniques that rely on available surface functional groups as grafting sites $[16,26]$. The mass of PEG that could be grafted to RO membranes was limited for large PEG polymers (MW 1,000 $\mathrm{g} \mathrm{mol}^{-1}$ ), with similar membrane properties observed for membranes modified with $1 \%$ and $15 \%$ PEG solutions [16]. For Jeffamine grafting to RO membranes, polymer chain length appeared to affect grafting density. Larger polymer chains were theorized to exert a steric effect that repulsed other polymer chains and resulted in a lower grafting density compared to the density of smaller Jeffamine polymer chains [26]. For the EDC-NHS mediated Jeffamine modifications in this work, confocal microscopy images combined with TBO dye test results suggest that not all carboxylic groups on the membrane surface have been grafted with Jeffamine, resulting in non-uniform, low-density surface coverage. The unreacted sites may represent surface carboxylic groups that are inherently inaccessible in the polyamide layer, which is estimated to be $20-60 \%$ of carboxylic groups [38], or these carboxyl groups may have been shielded from Jeffamine grafting because of a steric effect from adjacent grafted Jeffamine polymers.

\subsubsection{Surface roughness}

Surface roughness measurements for the $2 \%$ Jeffamine modified membranes and unmodified control membranes also demonstrate the non-uniformity of Jeffamine attachment. The surface roughness for $2 \%$ Jeffamine membranes, represented by root mean square roughness $(R M S, \mathrm{~nm})$, is significantly greater than the roughness of unmodified control membranes (twosided t-test, $\alpha=0.05$ ), as shown in Figure 6B. $R M S$ roughness is the square root of the mean of squared roughness values measured for each $100 \mu \mathrm{m}^{2}$ measurement area, and it represents the deviation of surface peaks and valleys from the mean height plane [39].

Increases in surface roughness have been reported for other post-fabrication PEG membrane modifications $[16,19,26]$, though a dependence on the initial roughness of the unmodified membrane surface has also been observed [18]. Increased surface roughness was also observed for PEG grafting to acyl chloride groups in the nascent polyamide layer of RO membranes during fabrication [11]. However, using a similar technique to modify polyamide FO membranes in situ resulted in a decrease in membrane surface roughness [12]. The different 
reported effects of PEG modifications on membrane surface roughness may be interpreted as a function of PEG grafting density. When high density is achieved, PEG chains form a uniform polymer brush that diminishes surface roughness features. However, low-density or nonuniform grafting causes localized areas of increased height that exaggerate overall surface roughness. For the $2 \%$ Jeffamine-modified membranes, the increase in surface roughness compared to the control membranes is attributed to the non-uniform Jeffamine attachment that was observed by fluorescence microscopy. Where micrometer-scale areas of relatively dense Jeffamine grafting occurred, local peaks were created that increased the RMS roughness.

\subsection{Fouling propensity of Jeffamine-modified membranes}

\subsubsection{Permeate flux decline due to organic fouling}

Flux decline in the organic fouling experiments occurs due to both the accumulation of foulants on the membrane surface and the draw solution dilution and feed solution concentration that result from batch operation of the FO test system. The accumulated foulant cake layer creates a physical resistance to water permeation as well as decreasing the driving force for permeation through cake-enhanced osmotic pressure [3]. Permeate flux decline in the baseline portion of the fouling experiment is attributed only to the effects of batch mode operation, and it enables the contribution of organic fouling to flux decline observed in the subsequent fouling portion of the experiment to be uniquely identified. For each organic fouling experiment, the flux decline due to organic fouling after $500 \mathrm{~mL}$ of permeate $\left(F D_{500 m L}, \%\right)$ was calculated as

$$
F D_{500 m L}=\frac{\left|\left(\frac{J_{W, f}}{J_{w, 0}}\right)_{\text {baseline }}-\left(\frac{J_{w, f}}{J_{w, 0}}\right)_{\text {fouling }}\right|}{\left(\frac{J_{W_{,} f}}{J_{W, 0}}\right)_{\text {baseline }}}(100) \quad \text { Eq. } 4
$$

where $J_{w, 0}$ is the initial water flux, and $J_{w, f}$ is the final water flux after $500 \mathrm{~mL}$ permeate has been collected. $F D_{500 m L}$ ranges from $0-100 \%$ where $0 \%$ represents no observed difference between flux decline in the baseline and fouling experiments, and thus no flux decline due to organic 
fouling. An $F D_{500 m L}$ value of $100 \%$ represents complete loss of permeate flux in the fouling experiment.

Jeffamine-modified membranes experience less flux decline due to organic fouling compared to unmodified control membranes when treating a mixed foulant feed solution, as shown in Figure 7. With $\mathrm{NaCl}$ draw solution, the mean $F D_{500 m L}$ for Jeffamine-modified membranes is $11 \%$ compared to $24 \%$ for the control membrane. For experiments using $\mathrm{MgCl}_{2}$ draw solution, mean $F D_{500 m L}$ values for Jeffamine-modified membranes and control membranes are $9 \%$ and $27 \%$, respectively. Complete permeate flux curves for the baseline, fouling, and recovery portions of each fouling experiment and the calculated $F D_{500 m L}$ values are included in Figures A1 and A2 and Tables A1 and A2 of Appendix A.

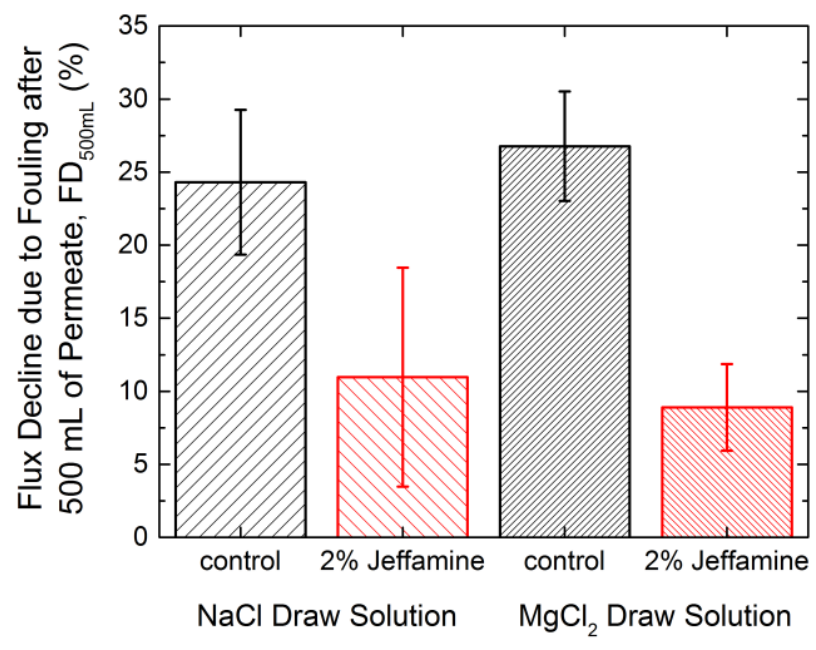

Fig. 7. Comparison of permeate flux decline due to fouling after $500 \mathrm{~mL}$ of permeate is collected $\left(F D_{500 m L}, \%\right)$ for unmodified control membranes and $2 \%$ Jeffamine modified membranes. $F D_{500 m L}$ values are shown for organic fouling experiments conducted with sodium chloride $(\mathrm{NaCl})$ draw solution (control $n=2$, modified $n=3$, initial baseline flux $J_{w, 0}=20.1 \pm 0.8 \mathrm{~L} \mathrm{~m}^{-2} \mathrm{~h}^{1}$ ) and with magnesium chloride $\left(\mathrm{MgCl}_{2}\right)$ draw solution (control $\mathrm{n}=3$, modified $\mathrm{n}=2$, initial baseline flux $\mathrm{J}_{\mathrm{w}, 0}=17.5 \pm 1.8 \mathrm{~L} \mathrm{~m}^{-2} \mathrm{~h}^{-1}$ ). Bars indicate the mean $F D_{500 \mathrm{~mL}}$ value, and error bars extend to one standard deviation. Foulant feed solution comprised $100 \mathrm{mg} \mathrm{L}^{-1}$ each of bovine serum albumin protein, sodium alginate, and Suwannee River natural organic matter dissolved into a synthetic secondary wastewater effluent solution $(0.94 \mathrm{mM}$ ammonium chloride, $0.45 \mathrm{mM}$ potassium phosphate, $0.5 \mathrm{mM}$ calcium chloride, $0.5 \mathrm{mM}$ sodium bicarbonate, $2.0 \mathrm{mM} \mathrm{NaCl}$, and $0.6 \mathrm{mM} \mathrm{MgSO}_{4}$ ) at solution $\mathrm{pH}$ 7.4 . 
The improved resistance to organic fouling for the Jeffamine-modified membranes indicates that the steric repulsion effect of the grafted Jeffamine has effectively reduced the attachment of foulants to the membrane surfaces. The corresponding resistance to permeation from an accumulated foulant layer was reduced compared to the unmodified control membranes, and any cake-enhanced osmotic pressure associated with the fouling layer was also reduced. Thus, higher permeate water fluxes were maintained throughout the fouling experiments.

Commercial NF and RO membranes that were modified with PEG and PEG derivatives also showed reduced water flux decline compared to unmodified control membranes when exposed to model foulants, and they were able to recover more of the initial permeate water flux after cleaning than control membranes $[16,19,26]$. Our previous work with FO membranes showed that the fouling propensity of membranes modified with PEG derivatives was reduced compared to unmodified control membranes [12,14], as discussed further in Section 3.4.

Unmodified control membranes demonstrated a higher mean $F D_{500 m L}$ during fouling experiments conducted with $\mathrm{MgCl}_{2}$ draw solution than during experiments with $\mathrm{NaCl}$ draw solution. Conversely, the Jeffamine-modified membranes exhibit lower mean $F D_{500 m L}$ when $\mathrm{MgCl}_{2}$ is used as the draw solution instead of $\mathrm{NaCl}$. The different fouling trends for control and $2 \%$ Jeffamine membranes are likely the result of different initial water fluxes for the experiments with $\mathrm{NaCl}$ and $\mathrm{MgCl}_{2}$ draw solutions. Previous work has demonstrated that the initial permeate flux affects the progression of organic fouling [40], especially for BSA protein [32], which was also used in this study.

After cleaning with $\mathrm{NaCl}$ solution at high crossflow velocity, both control and Jeffaminemodified membranes recover more than $85 \%$ of the initial permeate flux of the baseline condition. Calculated permeate water flux recoveries for the organic fouling experiments are included in Tables A1 and A2 of Appendix A. Fouling reversibility in FO is well-documented and is attributed to the relatively loose structure of the accumulated foulant layer [1]. The shapes of the flux decline curves for baseline, fouling, and recovery portions of each fouling experiment provide additional insight into the membrane fouling behavior. For the unmodified control membranes, the permeate flux decline curves for the recovery portions of the experiments (recovery curves) generally match the shapes and slopes of the flux decline curves for the fouling portions of the corresponding experiments (fouling curves). However, for the $2 \%$ Jeffamine 
membranes, the recovery curves generally display the same slopes as the flux decline curves for the baseline portions of the corresponding experiments (baseline curves). The matching slopes of recovery and fouling curves for control membranes suggest that these membrane surfaces were not completely cleaned and restored to their pristine condition during the physical cleaning portion of the experiments, regardless of the initial flux recoveries that were demonstrated. The surfaces of fouled control membranes may remain conditioned with foulants after cleaning, such that during recovery testing, permeate fluxes decline at rates equivalent to fouling testing conditions. For the $2 \%$ Jeffamine membranes, the opposite condition is indicated. Matching slopes of recovery and baseline curves suggest that organic foulants were completely removed from the modified membrane surfaces, and in the recovery testing, the membranes behave as they did in the baseline testing prior to their exposure to foulants.

\subsubsection{Surface adhesion forces}

AFM surface adhesion forces have been strongly correlated to membrane organic fouling behavior [41], and thus, AFM measurements were collected to elucidate the mechanisms of organic fouling resistance for the modified membranes. A maximum adhesion force $\left(F_{\max }\right)$ was calculated for each AFM probe retraction event, and the corresponding distance from the membrane surface was identified. The surface interaction energy between the foulant probe and the membrane surface was approximated by dividing $F_{\max }$ by the radius of the probe colloidal particle $\left(R_{p}\right)$. The quantity $F_{\max } / R_{p}$ is proportional to the surface interaction energy in accordance with the Derjaguin approximation [42].

Some AFM measurements exhibited no interaction energy, which was defined as one of three conditions: (i) probe retraction events where $F_{\max } / R_{p}$ was greater than zero, indicating repulsion from the membrane surface; (ii) probe retraction events where the absolute value of $F_{\text {max }} / R_{p}$ was less than $0.05 \mathrm{mN} \mathrm{m}^{-1}$, which is within the range of measurement noise; and (iii) probe retraction events where the absolute value of $F_{\max } / R_{p}$ was less than $0.10 \mathrm{mN} \mathrm{m}^{-1}$ at an associated distance greater than $300 \mathrm{~nm}$, which is also within the range of the AFM measurement noise. For the unmodified control membranes, surface interaction energies were approximated for 406 measurement events, and eight "no interaction energy" events were identified. For the 2\% Jeffamine membranes, 281 measurement events yielded 37 "no interaction energy" events. 
The mean and median values of surface interaction energies (excluding "no interaction energy" events) for the $2 \%$ Jeffamine membranes are $-0.32 \mathrm{mN} \mathrm{m}^{-1}$ and $-0.18 \mathrm{mN} \mathrm{m}^{-1}$, respectively. These interaction energies are not significantly different from the mean and median values of $-0.32 \mathrm{mN} \mathrm{m}^{-1}$ and $-0.21 \mathrm{mN} \mathrm{m}^{-1}$ for the control membranes. However, the Jeffaminemodified membranes exhibit an increase in "no interaction energy" events compared to the unmodified control membranes. The observance of equivalent measured interaction energies with an increased number of "no interaction energy" events for the Jeffamine-modified membrane is attributed to the non-uniform distribution of Jeffamine on the modified membrane surface. When the AFM probe contacted a Jeffamine-grafted area on the membrane surface, the probe experienced little attractive force or even repulsion from the surface due to the steric repulsion effect of Jeffamine polymer chains. In areas of the membrane surface without grafted Jeffamine, the AFM probe was essentially interacting with an unmodified membrane surface. Some "no interaction energy" events were also observed for unmodified control membranes, and these measurements may indicate less reactive areas of the membrane surface with low functional group density, as membrane surface chemistry and morphology are known to be heterogeneous $[43,44]$.

FO membranes modified with PEG derivatives in our previous work $[12,14]$ showed a decrease in the mean surface interaction energy between an AFM foulant probe and the membrane surface and also demonstrated an increase in the number of "no interaction energy" events. These membranes were modified in situ, during the membrane fabrication process. The reduced surface interaction energies for the in situ-modified membranes are a further indication that a higher density of PEG surface grafting was achieved via these in situ methods compared to this post-fabrication modification method.

\subsection{Performance of Jeffamine modification compared to other surface modification methods}

Though FO membranes modified post-fabrication with the EDC-NHS-Jeffamine method demonstrate reduced organic fouling, the fouling resistance appears to be limited by the density and uniformity of Jeffamine grafted to the membrane surface. Other FO membrane modification techniques demonstrated in our research group have achieved even greater organic fouling

resistance than this post-fabrication method, as demonstrated by reduced water flux decline due 
to fouling and reduced surface interaction energy for the modified membrane surfaces. These previously-demonstrated techniques include enriching the membrane surface with reactive sites during fabrication, followed by PEGylation [14]; incorporating PEG derivatives into the membrane active layer in situ during membrane fabrication [12]; and shielding the surface of the membrane with relatively large hydrophilic functionalized nanoparticles bound to the surface after fabrication [45]. The effectiveness of each of these modification techniques was assessed through dynamic organic fouling experiments similar to those described in this work, and $F D_{500 m L}$ values were calculated for each. The $F D_{500 m L}$ values are compared in Figure 8.

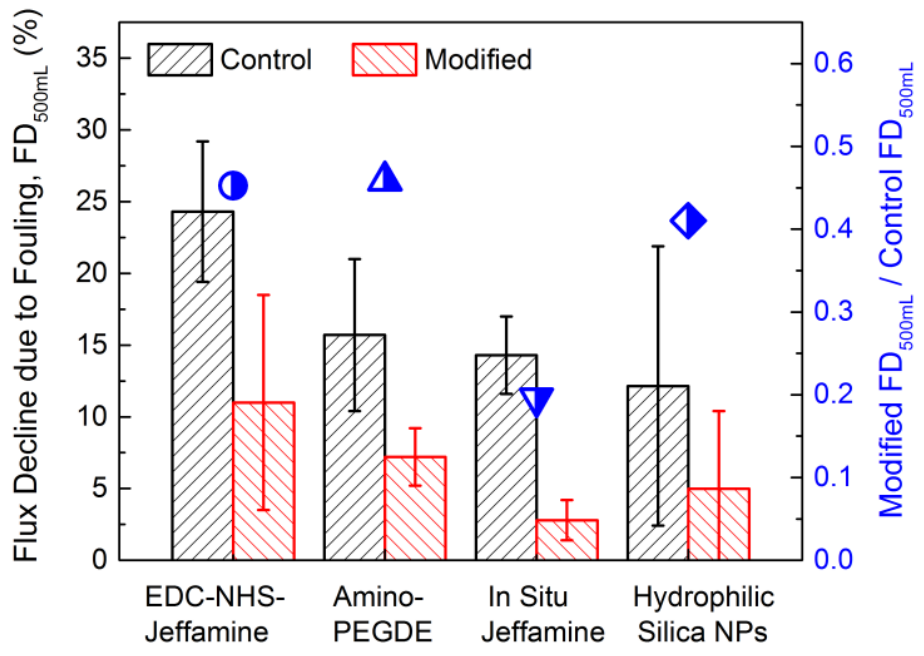

Fig. 8. Comparison of the organic fouling propensity of Jeffamine-modified membranes presented in this work to the fouling propensity of thin-film composite forward osmosis membranes modified using other techniques. The flux decline due to fouling after $500 \mathrm{~mL}$ of permeate is collected $\left(F D_{500 \mathrm{~mL}}, \%\right)$ using a sodium chloride draw solution is shown on the left vertical axis for unmodified control membranes and membranes modified using four different techniques: post-fabrication Jeffamine grafting technique that is presented in this work (EDC-NHS-Jeffamine, control $n=2$, modified $n=3$ ) with a mixed foulant feed solution containing $100 \mathrm{mg} \mathrm{L}^{-1}$ each of bovine serum albumin protein (BSA), sodium alginate, and Suwannee River natural organic matter (SRNOM); amine enrichment of the membrane surface followed by poly(ethylene glycol) diglycidyl ether attachment [14] (Amino-PEGDE, control $n=2$, modified $n=2$ ) with a foulant feed solution containing $250 \mathrm{mg} \mathrm{L}^{-1}$ sodium alginate; in situ modification with Jeffamine during the membrane fabrication process [12] (In Situ Jeffamine, control $n=3$, modified $n=3$ ) with a foulant feed solution containing $250 \mathrm{mg} \mathrm{L}^{-1}$ sodium alginate; and surface binding of superhydrophilic silica nanoparticles [45] 
(Hydrophilic Silica NPs, control $n=6$, modified $n=6$ ) with three different fouling feed solutions, each containing $150 \mathrm{mg} \mathrm{L}^{-1}$ of either BSA, sodium alginate, or SRNOM. Bars represent the mean $F D_{500 m L}$, and error bars extend to one standard deviation. The ratios of $F D_{500 m L}$ for the modified and control membranes of each FO membrane modification technique are represented by blue symbols that correspond to the right vertical axis.

Comparison of these different FO membrane modification techniques is made with an understanding that each technique was demonstrated with membranes from different sources using different feed solution foulants at different concentrations. Thus, $F D_{500 m L}$ values serve primarily as qualitative indicators of the effectiveness of each technique. However, the ratios of $F D_{500 m L}$ for the modified membrane to $F D_{500 m L}$ for the control membrane for each individual study provide a metric that is more useful for direct comparison. This ratio, shown on the right vertical axis in Figure 8, represents the fouling resistance of each membrane modification method for the conditions in which it was tested. FO membranes modified with the in situ Jeffamine technique show the greatest resistance to organic fouling, which may be a result of the relatively high Jeffamine grafting density that was achieved by performing PEGylation during fabrication. In situ membrane modification techniques require changes in the membrane fabrication process and may be difficult and expensive to implement at a manufacturing scale. The advantages of post-fabrication Jeffamine modification over the other FO modification techniques are its relative ease and low cost of implementation and its applicability to all types of commercial polyamide membranes.

\section{Conclusion}

The post-fabrication modification of TFC-FO membranes with PEG block copolymer to improve organic fouling resistance is accomplished via a simple, two-step grafting reaction. We identify $2 \%$ Jeffamine as the optimal solution concentration for this modification but observe that non-uniform Jeffamine grafting may be limiting the effectiveness of the modification. Additional optimization of grafting reaction conditions, such as EDC and NHS reaction concentrations and temperatures, may improve grafting uniformity and density for improved fouling resistance. Compared to previous work with FO membrane PEGylation, it appears that 
higher grafting density and fouling resistance may be achieved during fabrication, though with greater complexity and effort.

The study of different Jeffamine solution concentrations for modification highlights the tradeoff between improved fouling resistance with PEGylation and reduced membrane water permeability. For the treatment of high fouling potential feed waters, which is emerging as a niche commercial application of FO, a less permeable FO membrane may be an acceptable concession for the benefit of improved fouling resistance. The EDC-NHS mediated grafting technique demonstrated in this work may also serve as a useful platform for other TFC-FO membrane modification strategies, such as attachment of antifouling zwitterionic polymers or antimicrobial nanomaterials.

\section{Acknowledgements}

Financial support from the Department of Defense through the Strategic Environmental Research and Development Program (SERDP, Project ER-2217) is gratefully acknowledged. We also acknowledge a STAR Fellowship awarded by the US Environmental Protection Agency to D.L.S., a Graduate Research Fellowship awarded by the US National Science Foundation to H.J., and a graduate fellowship awarded by the China Scholarship Council to X.L. Facilities use was supported by YINQE and NSF MRSEC DMR 1119826. We thank Oasys Water, Inc. for providing the TFC-FO membrane samples.

\section{References}

[1] D.L. Shaffer, J.R. Werber, H. Jaramillo, S. Lin, M. Elimelech, Forward osmosis: Where are we now?, Desalination, 356 (2015) 271-284.

[2] B.D. Coday, P. Xu, E.G. Beaudry, J. Herron, K. Lampi, N.T. Hancock, T.Y. Cath, The sweet spot of forward osmosis: Treatment of produced water, drilling wastewater, and other complex and difficult liquid streams, Desalination, 333 (2014) 23-35.

[3] S. Lee, C. Boo, M. Elimelech, S. Hong, Comparison of fouling behavior in forward osmosis (FO) and reverse osmosis (RO), Journal of Membrane Science, 365 (2010) 34-39.

[4] Y. Kim, M. Elimelech, H.K. Shon, S. Hong, Combined organic and colloidal fouling in forward osmosis: Fouling reversibility and the role of applied pressure, Journal of Membrane Science, 460 (2014) 206-212. 
[5] M. Elimelech, W.A. Phillip, The Future of Seawater Desalination: Energy, Technology, and the Environment, Science, 333 (2011) 712-717.

[6] D. Rana, T. Matsuura, Surface modifications for antifouling membranes, Chemical reviews, 110 (2010) 2448-2471.

[7] S.I. Jeon, J.H. Lee, J.D. Andrade, P.G. De Gennes, Protein — surface interactions in the presence of polyethylene oxide: I. Simplified theory, Journal of Colloid and Interface Science, 142 (1991) 149-158.

[8] Z. Yang, J.A. Galloway, H. Yu, Protein Interactions with Poly(ethylene glycol) SelfAssembled Monolayers on Glass Substrates: Diffusion and Adsorption, Langmuir, 15 (1999) 8405-8411.

[9] S. Chen, L. Li, C. Zhao, J. Zheng, Surface hydration: Principles and applications toward lowfouling/nonfouling biomaterials, Polymer, 51 (2010) 5283-5293.

[10] K. Knop, R. Hoogenboom, D. Fischer, U.S. Schubert, Poly(ethylene glycol) in Drug Delivery: Pros and Cons as Well as Potential Alternatives, Angewandte Chemie International Edition, 49 (2010) 6288-6308.

[11] G. Kang, M. Liu, B. Lin, Y. Cao, Q. Yuan, A novel method of surface modification on thinfilm composite reverse osmosis membrane by grafting poly(ethylene glycol), Polymer, 48 (2007) $1165-1170$.

[12] X. Lu, S. Romero-Vargas Castrillón, D.L. Shaffer, J. Ma, M. Elimelech, In Situ Surface Chemical Modification of Thin-Film Composite Forward Osmosis Membranes for Enhanced Organic Fouling Resistance, Environmental Science \& Technology, 47 (2013) 12219-12228.

[13] H. Zou, Y. Jin, J. Yang, H. Dai, X. Yu, J. Xu, Synthesis and characterization of thin film composite reverse osmosis membranes via novel interfacial polymerization approach, Separation and Purification Technology, 72 (2010) 256-262.

[14] S. Romero-Vargas Castrillón, X. Lu, D.L. Shaffer, M. Elimelech, Amine enrichment and poly(ethylene glycol) (PEG) surface modification of thin-film composite forward osmosis membranes for organic fouling control, Journal of Membrane Science, 450 (2014) 331-339.

[15] A.C. Sagle, E.M. Van Wagner, H. Ju, B.D. McCloskey, B.D. Freeman, M.M. Sharma, PEGcoated reverse osmosis membranes: Desalination properties and fouling resistance, Journal of Membrane Science, 340 (2009) 92-108.

[16] E.M. Van Wagner, A.C. Sagle, M.M. Sharma, Y.-H. La, B.D. Freeman, Surface modification of commercial polyamide desalination membranes using poly(ethylene glycol) diglycidyl ether to enhance membrane fouling resistance, Journal of Membrane Science, 367 (2011) 273-287.

[17] S. Belfer, Y. Purinson, R. Fainshtein, Y. Radchenko, O. Kedem, Surface modification of commercial composite polyamide reverse osmosis membranes, Journal of Membrane Science, 139 (1998) 175-181.

[18] V. Freger, J. Gilron, S. Belfer, TFC polyamide membranes modified by grafting of hydrophilic polymers: an FT-IR/AFM/TEM study, Journal of Membrane Science, 209 (2002) 283-292. 
[19] L. Zou, I. Vidalis, D. Steele, A. Michelmore, S.P. Low, J.Q.J.C. Verberk, Surface hydrophilic modification of RO membranes by plasma polymerization for low organic fouling, Journal of Membrane Science, 369 (2011) 420-428.

[20] G. Ozaydin-Ince, A. Matin, Z. Khan, S.M.J. Zaidi, K.K. Gleason, Surface modification of reverse osmosis desalination membranes by thin-film coatings deposited by initiated chemical vapor deposition, Thin Solid Films, 539 (2013) 181-187.

[21] A. Matin, H.Z. Shafi, Z. Khan, M. Khaled, R. Yang, K. Gleason, F. Rehman, Surface modification of seawater desalination reverse osmosis membranes: Characterization studies \& performance evaluation, Desalination, 343 (2014) 128-139.

[22] Z. Grabarek, J. Gergely, Zero-length crosslinking procedure with the use of active esters, Analytical Biochemistry, 185 (1990) 131-135.

[23] J.V. Staros, R.W. Wright, D.M. Swingle, Enhancement by N-hydroxysulfosuccinimide of water-soluble carbodiimide-mediated coupling reactions, Analytical Biochemistry, 156 (1986) 220-222.

[24] A. Tiraferri, C.D. Vecitis, M. Elimelech, Covalent Binding of Single-Walled Carbon Nanotubes to Polyamide Membranes for Antimicrobial Surface Properties, ACS Applied Materials \& Interfaces, 3 (2011) 2869-2877.

[25] F. Perreault, M.E. Tousley, M. Elimelech, Thin-Film Composite Polyamide Membranes Functionalized with Biocidal Graphene Oxide Nanosheets, Environmental Science \& Technology Letters, 1 (2013) 71-76.

[26] G. Kang, H. Yu, Z. Liu, Y. Cao, Surface modification of a commercial thin film composite polyamide reverse osmosis membrane by carbodiimide-induced grafting with poly(ethylene glycol) derivatives, Desalination, 275 (2011) 252-259.

[27] D. Saeki, S. Nagao, I. Sawada, Y. Ohmukai, T. Maruyama, H. Matsuyama, Development of antibacterial polyamide reverse osmosis membrane modified with a covalently immobilized enzyme, Journal of Membrane Science, 428 (2013) 403-409.

[28] Huntsman, Jeffamine(R) ED-2003 Polyetheramine Technical Bulletin 5197-0208, Huntsman Corporation.

[29] D. Sehgal, I.K. Vijay, A Method for the High Efficiency of Water-Soluble CarbodiimideMediated Amidation, Analytical Biochemistry, 218 (1994) 87-91.

[30] M. Mulder, Basic Principles of Membrane Technology, Second ed., Kluwer Academic Publishers, 1996.

[31] A. Tiraferri, M. Elimelech, Direct quantification of negatively charged functional groups on membrane surfaces, Journal of Membrane Science, 389 (2012) 499-508.

[32] B. Mi, M. Elimelech, Chemical and physical aspects of organic fouling of forward osmosis membranes, Journal of Membrane Science, 320 (2008) 292-302.

[33] M. Herzberg, M. Elimelech, Biofouling of reverse osmosis membranes: Role of biofilmenhanced osmotic pressure, Journal of Membrane Science, 295 (2007) 11-20.

[34] G.-d. Kang, Y.-m. Cao, Development of antifouling reverse osmosis membranes for water treatment: A review, Water Research, 46 (2012) 584-600. 
[35] C.Y. Tang, Y.-N. Kwon, J.O. Leckie, Probing the nano- and micro-scales of reverse osmosis membranes - A comprehensive characterization of physiochemical properties of uncoated and coated membranes by XPS, TEM, ATR-FTIR, and streaming potential measurements, Journal of Membrane Science, 287 (2007) 146-156.

[36] Y.-N. Kwon, J.O. Leckie, Hypochlorite degradation of crosslinked polyamide membranes: II. Changes in hydrogen bonding behavior and performance, Journal of Membrane Science, 282 (2006) 456-464.

[37] I. Noda, A. Dowrey, J. Haynes, C. Marcott, Group frequency assignments for major infrared bands observed in common synthetic polymers, in: Physical Properties of Polymers Handbook, Springer, 2007, pp. 395-406.

[38] O. Coronell, M.I. González, B.J. Mariñas, D.G. Cahill, Ionization Behavior, Stoichiometry of Association, and Accessibility of Functional Groups in the Active Layers of Reverse Osmosis and Nanofiltration Membranes, Environmental Science \& Technology, 44 (2010) 6808-6814.

[39] ISO, 4287: Geometrical Product Specifications (GPS)-Surface texture: Profile methodTerms, definitions and surface texture parameters, International Organization for Standardization, 1997.

[40] C.Y. Tang, Q. She, W.C.L. Lay, R. Wang, A.G. Fane, Coupled effects of internal concentration polarization and fouling on flux behavior of forward osmosis membranes during humic acid filtration, Journal of Membrane Science, 354 (2010) 123-133.

[41] S. Lee, M. Elimelech, Relating Organic Fouling of Reverse Osmosis Membranes to Intermolecular Adhesion Forces, Environmental Science \& Technology, 40 (2006) 980-987.

[42] J.N. Israelachvili, 11 - Contrasts between Intermolecular, Interparticle, and Intersurface Forces, in: J.N. Israelachvili (Ed.) Intermolecular and Surface Forces (Third Edition), Academic Press, San Diego, 2011, pp. 205-222.

[43] V. Freger, Nanoscale Heterogeneity of Polyamide Membranes Formed by Interfacial Polymerization, Langmuir, 19 (2003) 4791-4797.

[44] F.A. Pacheco, I. Pinnau, M. Reinhard, J.O. Leckie, Characterization of isolated polyamide thin films of RO and NF membranes using novel TEM techniques, Journal of Membrane Science, 358 (2010) 51-59.

[45] A. Tiraferri, Y. Kang, E.P. Giannelis, M. Elimelech, Superhydrophilic Thin-Film Composite Forward Osmosis Membranes for Organic Fouling Control: Fouling Behavior and Antifouling Mechanisms, Environmental Science \& Technology, 46 (2012) 11135-11144. 


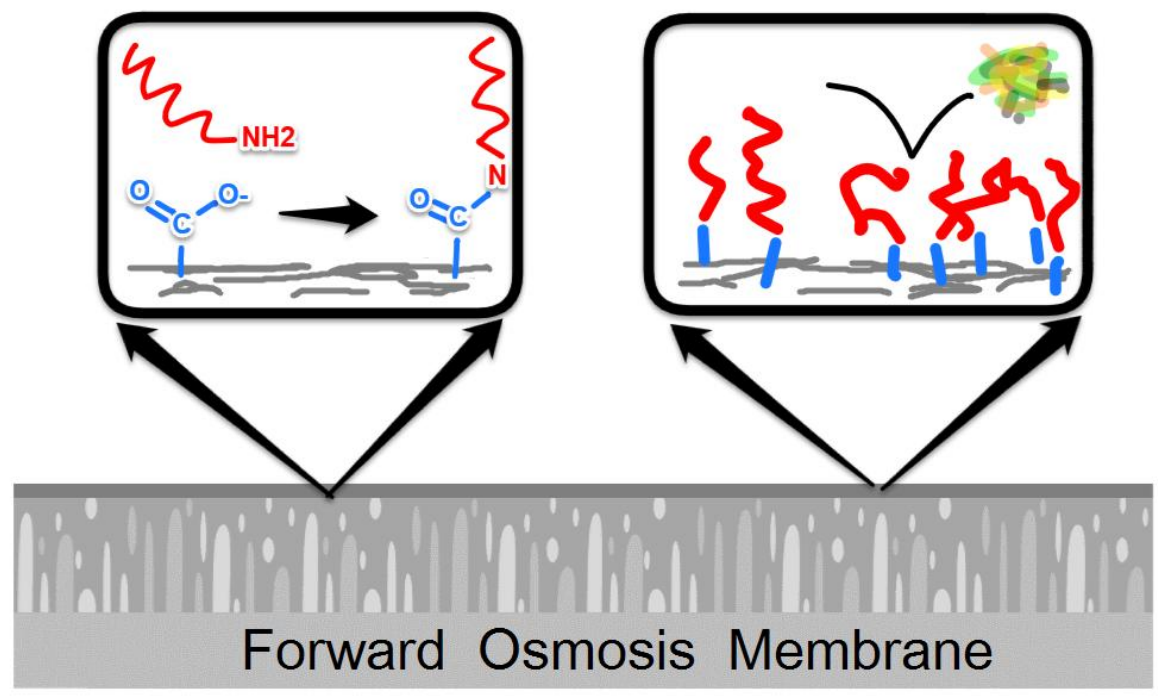

